\title{
What Price Changing Laws of Nature?
}

\section{Forthcoming in the European Journal for Philosophy of Science}

Olivier Sartenaer [corresponding author]

Institut supérieur de Philosophie, Université catholique de Louvain, Belgium

olivier.sartenaer@uclouvain.be

\author{
Alexandre Guay \\ Institut supérieur de Philosophie, Université catholique de Louvain, Belgium \\ Paul Humphreys \\ Corcoran Department of Philosophy, University of Virginia, USA
}

\begin{abstract}
In this paper, we show that it is not a conceptual truth about laws of nature that they are immutable (though we are happy to leave it as an open empirical question whether they do actually change once in a while). In order to do so, we survey three popular accounts of lawhood -(Armstrong-style) necessitarianism, (Bird-style) dispositionalism and (Lewis-style) 'best system analysis' - and expose the extent, as well as the philosophical cost, of the amendments that should be enforced in order to leave room for the possibility of changing laws.
\end{abstract}

\section{Acknowledgments}

We would like to thank Michael Hicks, Vera Hoffmann-Kolss, Andreas Hüttemann and Christian Loew for helpful comments and/or discussions on earlier versions of (parts of) this paper, as well as the audiences of the Köln Kolloquium Wissenschaftstheorie, the Third International Conference of the German Society for Philosophy of Science (GWP) and the Francqui Chair lectures at KU Leuven, where parts of this paper have been presented. Olivier Sartenaer gratefully acknowledges the financial support of the Alexander von Humboldt Foundation and Paul Humphreys would like to acknowledge a 3 Cavaliers grant from the University of Virginia. 


\section{Introduction}

'They were wrong and he was right. The obvious, the silly, and the true had got to be defended. Truisms are true, hold onto that! The solid world exists, its laws do not change. Stones are hard, water is wet, objects unsupported fall toward the earth's centre.'

(George Orwell, 1984)

Cynics sometimes have it that philosophical statements are either truistic or false. In this paper-and perhaps in a way that would conveniently put us in the good graces of Orwell's Thinkpol-we show that it is not a conceptual truth about laws that they are immutable. As relatively uncynical philosophers, though, we are happy to leave it as an open empirical question whether they do actually change once in a while.

Apart from some exceptions (Poincaré 1911; Whitehead 1933; or, more recently, Balashov 1992; Shimony 1999; Lange 2009; Tahko 2015), the well-entrenched assumption that laws of nature are necessarily immutable has been seldom critically put into question. Yet, besides these philosophers' moral right to speculate off the beaten path, such an assumption has been challenged to different extents by practicing scientists (see, e.g., Dirac 1937; or, more recently, Smolin 2015). Though most of the time it is not perfectly clear what those scientists might exactly be committed to when they are claiming that laws of nature 'change' or 'evolve', the very fact that they happen to take this idea seriously - in spite of its controversial flavor-certainly is an indication that some philosophical work deserves to be done.

Obviously, whether or not laws of nature can change, as well as how precisely they may come to do that-if they do it at all-crucially depends on what the concept of 'law of nature' is taken to refer to, that is to say, what philosophical account of lawhood one holds dear. This relatively bland observation renders our already delicate endeavor even more hazardous from the outset, given the very wide diversity of ways in which the concept of a law of nature has been articulated by philosophers over the last decades. The least we can say is that when it comes to defining what laws of nature are, philosophers are not near to reaching (the beginning of) a consensus. This motivates our chosen methodology: in what follows, we undertake to remain 
somewhat neutral with respect to such a controversy by surveying three of the most popular theories of lawhood available and, for each of them, evaluating the extent to which they allow for accommodating the idea that laws of nature change. 1

More precisely, here is the plan. In section 1, and as a representative of those accounts of lawhood which rely on the idea that the nomological source of change in a world is external to the entities populating that world, we explore the possibility that laws of nature change within the framework of Armstrong's necessitarianism. We then turn in section 2 to dispositionalism, as the best available account of lawhood that considers the source of change as rather internal to worldly entities themselves. Finally, in section 3 , we deal with Humean regularity theories that eschew any commitment to (natural) necessity, with a focus on the mainstream, Lewis-style 'best system analysis'. In all of these frameworks, we settle on exposing the extent, as well as the philosophical cost, of the amendments that should be enforced in order to leave room for the possibility of changing laws.

\section{Changing Laws in Governism}

Governism is a metaphysical conception of natural law founded on the hypothesis that natural entities are subjected to change; that change is enacted upon them: in other words, that the metaphysical source of change is in a certain sense external to the entities that are changing. ${ }^{2}$ This external conception of change is partly grounded in the metaphysical assumption that it is possible to have a world with the same natural properties but different laws. In consequence, governism should be contrasted with positions where the metaphysical source of change is internal, such as dispositionalism and with positions where there are no necessary connections at all, such as Humeanism.

Such a position has been defended and developed by many philosophers. However, for the rest of this discussion, we will concentrate on Armstrong's (1983) conception of natural laws, for it is undeniably one of the clearest attempts to develop a governist conception of natural

\footnotetext{
1 In this respect, we share the spirit of a similar endeavor undertaken by Cartwright and Merlussi (2018) relative to the contingency that laws possibly allow.

2 In this paper we concentrate on dynamical laws. As in physics, we suppose these are more fundamental than the nondynamical laws.
} 
necessity. ${ }^{3}$ According to Armstrong, a law of nature is essentially a relation between universals that have natural properties as instances. Sentences like 'it is a law that Fs are Gs' should be understood as 'it is physically necessary that Fs are Gs'. It should be noted that the necessity involved here is weaker than logical necessity but is still a necessity, nonetheless. How does this necessity relate universals? Armstrong argued that all laws of nature are grounded on necessitation relations between universals. In the case that occupies us, we would represent this relation as $\mathrm{N}(\mathrm{F}, \mathrm{G})$. This relation refers to states of affairs that can be described as follows:

Something's being F necessitates that the same something's being G, in virtue of the universals F and G. (Armstrong 1983, 96)

In this context, would it be possible for a natural law to change? Before answering, we have to make the question clearer. What would be an appropriate conception of change here? It cannot be that the laws are apparently different here from there. Time should play a role. The laws should dynamically change. ${ }^{4}$ They should differ at different times. Moreover, we want to explore two quite different possibilities: local and global change of laws. The first possibility refers to the case where laws could change depending of the local context, the second to the case where laws change in all the universe at the same time. ${ }^{5}$ For example, if someone wants to argue that organic systems are not completely governed by physical laws, but nevertheless do not involve the introduction of new properties from outside, they could mean at least two things: (a) that, each instance, when matter is organized in organic systems, new laws appear and govern such a system (local version), or (b) at one point in time, new organic laws came about and are still governing organic systems (global version).

Trying to extend Armstrong's framework in the context of our question, we believe there are two configurations that could potentially be understood as a change of laws: (1) a system

\footnotetext{
3 There are other forms of governism that seem more compatible with changing laws, for example, natural laws as divine decrees or certain kinds of primitivism about laws. Since our goal is to challenge the assumption that laws cannot change, we decided to treat a kind of governism that is the less welcoming to changing laws, on this point see Hildebrand (2018).

${ }^{4}$ Of course, if one defends a static conception of change, it is maybe not a fatal flaw, even if defenders of the static conception of change usually distinguish spatial and temporal difference. We also put aside all complications about time that could come if the general theory of relativity is true.

5 This could remind the reader of the notion of 'cosmic epochs' discussed by Armstrong (1983).
} 
subjected to a change of necessities and (2) a system changing properties, but not in the expected way; not under the action of an already established natural law. Let us discuss each possibility.

\subsection{A System Subjected to a Change of Necessities}

Two situations, apparently involving the same properties, differ radically in their behavior. The simplest explanation is that they are not governed by the same laws, even if they should be since they exhibit the same properties. This scenario could be compatible with either the local or the global change conception. However, to show that this simple explanation is a genuine case is not easy. For example, let us imagine a situation $A$, that corresponds to a gas trapped in a closed box and a situation $B$, where the lid of the box is now open and the gas diffuses freely. Between $A$ and $\mathrm{B}$, the behavior of the gas is remarkably different, nevertheless we would not say that the laws have changed between A and $\mathrm{B}$. The same mechanical laws are acting, but the context is very different. Note that we could possibly built a function that will describe the correlation between the kinds of behavior and the kinds of context. Still, it would be understood by most physicists as an accidental regularity, not a law.

This thought experiment shows that in order to claim that it is the laws that have changed, we have to be able to distinguish laws from all other contextual elements; in other words, to distinguish between laws and initial or boundary conditions. We have to distinguish two sources of change: the dynamics (the laws) from the context (the initial conditions). In the literature, this distinction is far from being consensual. For example, for Wigner, it is essentially an epistemological distinction with no clear reference in reality (Wigner 1979, chapter 1); for Earman, it is an unclear presupposed distinction essential to understand the role of symmetries, the major concept of current theoretical physics (Earman 2004). In the context of governism, the distinction could be understood to be that laws are about necessitation connections between universals, whereas initial conditions are about all other accidental aspects. Unfortunately, this metaphysical distinction does not provide us with a useful practical criterion to distinguish laws from initial conditions. However, for the rest of this discussion, we will suppose that such distinction is possible. This said, let us continue our main line of thought.

There are two possible ways to understand the transition between nomological regimes in situation $A$ and situation $B$, both situations involving the same natural properties. The first is that it 
is a brute fact that is not explained by Armstrong's conception of laws. If the apparent nomological change happens only once, this is not worrisome. However, if it is regularly happening, it is a problem: remember governism is a metaphysical theory supposed to explain natural regularities. The second is that it is the result of a kind of necessity. The change of nomological regime would be the result of the action of a meta-law, a law governing this transition, noted as $N_{2}\left(L_{A}, L_{B}\right)$, where $L_{A}$ and $L_{B}$ are the laws governing situation $A$ and situation $B$ respectively. In Armstrong's framework, it is in virtue of $L_{A}$ and $L_{B}$ that the nomological transition is described by a necessitation relation. If this meta-law exhibits a different kind of necessity than natural laws, a meta-necessity, a difficulty follows. As mentioned above, in Armstrong's framework, necessitation relations are grounded in universals. In consequence, different kinds of universals should be associated with different kinds of necessity. For example, natural and mathematical necessities seem clearly different because natural and mathematical properties are different. However, in the current case, the meta-laws are invoked to explain the change laws; in other words, the change of behaviors that are themselves explained by first order laws. These symmetrical roles push us towards identifying both necessities. ${ }^{6}$ The alternative would be to say that the two Ns, regular and meta-necessity, are Ns inasmuch as they are instances of some other $\mathrm{N}_{3}$, a broader notion of necessity. What this third necessity would be is not clear to us.

So, it seems preferable to keep only one kind of necessity. If there is only one kind of $\mathrm{N}$ then the $L_{i}$ in $N\left(L_{A}, L_{B}\right)$ are not just symbols representing laws, they refer to universals, perhaps to natural properties, 'to be a law of such and such kind'. These properties are relational properties between basic natural properties. It is not clear to us how the domain of such properties could be defined and if they could still be called natural properties. Indeed, it is difficult to see how two different dynamical laws would or would not be in the same kind. 7

One of the ways to sustain such a position would be to weaken the notion of natural necessity. $\mathrm{N}$ would denote a contextual necessity. This seems to us costly, because a weak notion of necessity would drastically diminish the explanatory power of the governist account of natural laws and potentially would bring the many necessities puzzle back. The other possibility would be to weaken the universals. We could argue that $L_{A}$ and $L_{B}$ are not different universals associated with different nomological relations among the same universals. Rather, they are

\footnotetext{
6 A similar argument can be found in Yudell (2013).

7 Principles of symmetries when understood as meta-laws raise a similar difficulty.
} 
referring to changes among quasi-universals and therefore they are not genuine natural laws, but are contextual laws. This is not because they involve different necessities, but rather, because they involve quasi-universals. The potential genuine laws are the ones that relate the universals to which the quasi-universals refer.

But are quasi-universals even making sense in the governist approach? According to Armstrong, quasi-universals 'resemble universals permitting a multitude of instances, but unlike universals proper, they involve temporal or spatial restrictions' (Armstrong 1983, 77). Two kinds of situation are envisioned by Armstrong: (1) laws that differ depending on location (Smith's garden laws) and (2) laws that evolve through time (nomological epochs). As mentioned above, the first kind is not what we need for dynamical change of laws. As for the second kind, it seems appropriate to model global change but not local change. To go back to the above example about organic systems, what would be needed is a broader contextual notion of quasi-universal, e.g., physical properties involved in organic structures vs. physical properties involved in inorganic systems. To our knowledge, such a notion of indexical universals does not exist in the literature. The best, but limited attempt, to develop such a theory of indexical universals is in Maudlin (2007, chapter 3 ) and it is only a sketch. Let us be crystal clear about the cost. The development of a new theory of universals, an obviously difficult task, is a condition to produce a coherent conception of natural laws in this metaphysical framework. But what about the second possibility? We will show below that it will not simplify our problem.

\subsection{A System Changing Properties}

To capture the paradigmatic case of the second possibility of changing laws, let us imagine a system governed by the law $L_{A}$ and, at a later time, governed by the law $L_{B}$, with $L_{A} \neq$ $L_{B}$. Note that contrary to the previous section, it is not the same set of properties that are instantiated in situation A and situation B. Could this process be a genuine change of laws? Note that this case seems more compatible with the local conception, but it could be global if the system in question is the entire universe at some time. If the process involves a modification of the identity of the system, to the point where we could not say that the system governed by $L_{A}$ is the same as the one governed by $L_{B}$, then it seems hopeless to defend the position that we face a nomological change. It is a case analogical to the fact that different systems could be governed by different laws. Let us explore this further. 
On the other hand, if we have reasons to believe that the two situations concern the same system, we cannot dismiss immediately this case. Since situations A and B do not share as a hypothesis relevant properties, on what ground could we establish the system identity? If the identity of an entity depends upon the laws it obeys, as Newton argued (Brading 2013), this path is closed. In order to claim a continuity of the system identity, we need to disentangle between what it means to be such and such system and what it means to be a subject of a law.

A natural way to show that situation $A$ and situation $B$ are possibly about the same system is to argue that they are causally related, in an ontological fashion, and that this relation is solely, or at least mainly, responsible for the change of laws. Indeed, in this context, we would have reason to say that we are talking about the same system even if it instantiates different properties. Unfortunately, in the governist approach, a causal relation among particulars is generally understood as the result of the instantiation of a law. We would have to sustain that this nomological reduction of causality is only partial; that some causal links are not captured by normal laws, but by other kinds of causal necessity. As we have seen above, this multiplication of necessities is costly in the context of governism. Nevertheless, there are other means by which to establish a continuity of identity through property change: essence, bare individuality...but they all diminish significantly the elegant simplicity of the governist position. Moreover, even if we manage to develop such a position, we do not see how a skeptic could not simply assert that in this process laws did not change, only systems did.

In conclusion, we believe that we have shown that it is possible to allow changes of laws in the context of governism. However, this is not without a cost. The apparently cheapest option would be to develop the theory of natural laws in a framework where universals are indexical.

\section{Changing Laws in Dispositionalism}

Dispositionalism has in recent years most commonly been developed within the framework of dispositional essentialism, a position with the consequence that the manifestations of a disposition cannot, as a matter of metaphysical necessity, fail to appear when the appropriate stimulus conditions for the disposition are present. As a result, if a law is rooted in such dispositions, that law cannot be contingent.8 Although the contingent status of a law does not

\footnotetext{
8 More specifically, there are no worlds in which the dispositional property and its stimulus exist and cooccurrences of instances of them are not accompanied by an instance of the manifestation property. The following discussion does not depend on whether the relevant properties exist in all possible worlds or only in some worlds.
} 
entail that it can change over time, the argument given here removes a major obstacle to the possibility of changing laws. There are multiple variants of dispositional essentialism but we shall here take Bird (2007) as representative of the spirit of the dispositional essentialist approach to laws. We also restrict ourselves to fundamental laws and properties.

\subsection{Ontology}

Definition. A property $D$ is dispositional just in case $D$ would manifest characteristic properties when characteristic stimulus properties are present, and $D$ is also present at times when those stimulus conditions are absent. The property is therefore identical neither with its manifestations nor with its stimulus conditions. 9

A dispositional property has the generic form $D(a, S, M)$, where $a$ is a region of spacetime at which $S$ is instantiated, $S$ is a collection of stimulus properties, and $M$ is a collection of manifestation properties. $\mathrm{D}(\mathrm{a}, \mathrm{S}, \mathrm{M})$ is read as "a has the disposition to manifest $\mathrm{M}$ when conditions $S$ are present". The detailed nature of $D$ is abstracted from here to allow for a variety of views about the form of dispositions. The generic form is intended to capture causal or noncausal relations between the properties as well as the case where dispositional properties produce other dispositional properties. Those who prefer to deal in property instances can quantify over objects and times in the generic form. To avoid question-begging, $D(a, S, M)$ is not assumed to have a particular degree of modal strength, although at this point the possibility is left open that it might be metaphysical necessitation. For simplicity, the analysis is restricted to deterministic laws, so that the quantitative case $D$ is such that for any given values of the determining properties $S$, there are unique values of the determined properties $\mathrm{M}$.

The standard contrast to dispositional properties is categorical properties but that contrast is neither uniformly drawn nor entirely neutral with respect to the goals in this paper. For example, Bird $(2007,3)$ holds that fundamental categorical properties are those whose natures are picked out by quiddities and for which there are no necessary connections with other entities (op. cit. $2007,67)$. This dichotomy between categorical and dispositional properties is an uncomfortable

\footnotetext{
9 Tugby (2013) argues that not all dispositional properties need have stimulus conditions. We set aside such cases
} here. 
fit in the present context because fundamental laws may contain mixtures of dispositional and categorical properties. We shall therefore not appeal to that contrast here.

\subsection{Quantitative Laws}

Dispositional essentialism is the position that the identity conditions for a dispositional property result from an essence that the disposition possesses, where $E$ is an essence of property $P$ just in case necessarily, if property $Q$ lacks $E$, then $Q \neq P$. The essence of the dispositional property fragility, for example, is to produce a shattering when striking is present. There are grades of commitment to dispositional essentialism. The strongest grade of commitment, dispositional monism, held by Alexander Bird (2007, Chapter 2) and Sidney Shoemaker (1980), is that all properties, in the case of Bird all fundamental properties, have dispositional essences. We shall address that grade of commitment because it leads to the position that if fundamental laws are patterns that result from the manifestation of fundamental dispositional properties, then all fundamental laws are metaphysically necessary.

Can laws change over time under this view? We shall first argue that although some fundamental qualitative laws may be metaphysically necessary, at least some fundamental quantitative laws are not. Having shown that laws can change across possible worlds, we then go on to argue that laws may be temporally limited in a way similar to laws with limited spatial scope.

Dispositional essentialism holds that the fundamental properties have dispositions that are essential to that property, that is $\square(P(a) \rightarrow D(a, S, M))$. For, at least with respect to fundamental properties, those properties are individuated by their dispositional attributes and if the fundamental property failed to manifest its characteristic dispositional behavior it could not be the property it is held to be. If a putative positive charge $\mathrm{P}(\mathrm{a})$, when placed near a second positive charge $S(b)$, did not produce the characteristic manifestation of being repelled by the field produced by $S(b)$ when no other forces were present on a, the attribution to a of being positively charged would be incorrect. There thus cannot be a case in which the juxtaposition of two positive charges is not followed by their repelling one another, where the "cannot" is metaphysical necessitation.

Let us grant for the moment that the sentence "Positive charges repel other positive 
charges" is a metaphysically necessary truth on the grounds that anything that does not repel a positive charge ipso facto does not itself possess the property of being positively charged. But scientific laws, at least in physics, go beyond simple qualitative relations such as "Like charges repel one another". They involve quantitative relations and have a specific mathematical form. Here is a simple hypothetical example. Suppose that Hooke's Law is a fundamental law of world W. There are various versions of Hooke's Law; here we are taking the phenomenological version that states 'The extension of a spring or wire is directly proportional to the force applied'. The fundamental properties in W are of three kinds: the property of being a wire, the property of having a spatial extension, and the property of being a force.10 Wires in W are noncomposite objects, not being made up of more fundamental entities, and it is important that we can identify them in terms of features other than Hooke's Law. One way to do this is to identify them using the properties of being thin, being cylindrical, and being metal. This characterization still allows the property of being a wire to have a qualitative dispositional essence, in that the property of being a wire entails a qualitative disposition to extend when a force is applied along the direction of the wire. It is not a quantitative disposition characterized by Hooke's Law. On this basis, let us allow that, analogously to the qualitative form of Coulomb's Law, the qualitative law 'Wires extend when a longitudinal force is applied' is metaphysically necessary. ${ }^{11}$ However, this qualitative truth does not give us the mathematical form of the relation between the extension of the wire and the applied force. We can now consider a quantitative dispositional property possessed by wires, which is a disposition to extend by $f(x)$ under the application of a force F. In world W, the relation is linear and fundamental, where a fundamental relation is one for which there is no explanation. Thus, in W, F = kx for the force needed to extend by $\mathrm{x}$ a wire with modulus of elasticity k. ${ }^{12}$ Call this law L.

Suppose that in a second world, $\mathrm{W}^{*}, \mathrm{~F}=\mathrm{k}^{*} \mathrm{x}$, where $\mathrm{k} \neq \mathrm{k}^{*}$. Do we have the same law with a different coefficient or a different law? On a very strict interpretation of essentialism, one's inclination might be to argue that this is a different law because the dependent variable

\footnotetext{
10 To keep the prose simple, we shall from now on refer to 'wires' as shorthand for the instantiation of the property of being a wire.

${ }^{11}$ We are not endorsing this claim of metaphysical necessity, but allowing it for purposes of argument.

12 In world W, the modulus of elasticity for a given material is a fundamental constant. It is not the result of the internal composition of the material.
} 
changes by a different amount with a unit increase in the independent variable and so the wire in $\mathrm{W}^{*}$ follows a different regularity than it does in $\mathrm{W}$. But that inclination is not conclusive, for within our own world, we hold that wires with different values of the modulus of elasticity follow the same law, Hooke's Law, just with different coefficients. This response can be justified by noting that the values of $\mathrm{k}$ are not elements of a law but contextual elements, akin to initial conditions, and so are not responsible for changes in laws. ${ }^{13}$ So take a third world $\mathrm{W}^{\star \star}$ with the same fundamental entities where $\mathrm{f}$ is quadratic and fundamental so that the form of the law is $F=-k x^{2}$. Call that law $L^{\star *}$. $L^{\star *}$ should be considered to be a different law from $L$ because it has a different mathematical form, nonlinear as opposed to linear. This example is no longer about a change in a fundamental constant but about a change in the mathematical form of the law.

A determined defender of the metaphysical necessity of laws might maintain that in both $\mathrm{W}$ and in $\mathrm{W}^{\star \star}$ wires obey the same polynomial law, only with different degrees of the polynomial. But unless the defender of metaphysical necessity is willing to argue that all mathematical functions have a single underlying form, we can always find some other $\mathrm{W}+$ in which wires follow a different mathematical regularity. In both $\mathrm{W}$ and $\mathrm{W}+$, the wire has a disposition to require a force $f(x)$ when stretched by $x$ but the law covering that force has changed between W and W+. Hooke's Law, the quantitative law that holds in our world, is not essential to that disposition because wires, forces on wires and extensions of wires have identity conditions that are independent of Hooke's Law as given above. It is therefore not essential to wires that they conform to Hooke's Law across worlds. Again, a determined essentialist could maintain that the quantitative form of the law is essential to the disposition, but as Fine (2002) has noted, this is at odds with how we assess possibilities. Thus at least some fundamental laws are not metaphysically necessary.

Some earlier authors have argued for related conclusions. Hendry and Rowbottom (2009) argue for the contingency of natural laws but restrict themselves to small changes in the form of the law. Keinänen (2014) uses a trope and causal process ontology to argue for slight changes in the functional form of laws or changes in natural constants. In contrast, the argument given here allows for significant changes in the mathematical form of fundamental

\footnotetext{
${ }_{13}$ Bird $(2007,212-213)$ argues that historical evidence suggest that we can eventually explain why fundamental constants have the values that they do. In the example given here, however, it is a feature of $W^{*}$ that $k^{*}$ is fundamental. See also Lowe (2002).
} 
laws. Bird $(2007,48-49)$ on the other hand argues that all fundamental laws are metaphysically necessary on the grounds that a true account of fundamental laws should be unified. This is to impose a metaphysical tidyness principle on a world that may be fundamentally messy, a position that concedes too much to the best system account. It may be objected that dispositional essentialism asserts only that at least one property of a disposition is essential. We can accept that point and note that because the argument involving the mathematical form of laws generalizes widely, and most laws in fundamental sciences have a mathematical form, dispositional essentialism is probably confined to cases involving qualitative laws. That number is arguably non-zero, because the basic "Like charges repel" form of Coulomb's Law looks amenable to dispositional essentialism. This is because a central feature of the argument just given is that one can, and often does, qualitatively identify the properties involved in a law before the mathematical form of the law is discovered. ${ }^{14}$ What of the inverse square law that accompanies Coulomb's Law? Here symmetry considerations that underlie many inverse square forms when a geometrically homogeneous field is involved can be cited in favor of essentialism, but, along the lines of the point made earlier, this will be a required feature of a world only under a stipulative simplicity condition requiring homogeneity.

\subsection{Temporal Changes in Laws.}

To facilitate a transition between the contingency of laws and changes of laws over time, two kinds of necessity can be considered. In the first type of necessity, the contingent nomological necessity attached to laws of nature is reduced to a specification of at which other possible worlds the regularity associated with the law occurs, the number of such worlds lying between the full complement of worlds associated with metaphysical necessity and the much smaller number of worlds at which accidental occurrences of the regularity are present. This can be called the external account of necessity because the criteria for its presence involve worlds external to our own. ${ }^{15}$ In contrast, the internal account of necessity involves a relation of necessitation within our world. Its canonical example is that of a non-

\footnotetext{
14 The situation is complicated by the fact that Coulomb's Law is often considered not to be a fundamental law but a consequence of the more general Gauss's Law.

15 In the possible worlds approach it seems to be an implicit assumption that the laws of a given world are fixed, although it would be easy to adapt the approach to relax that assumption.
} 
Humean causal necessitation in which a cause necessitates the occurrence of its effect. To put the distinction starkly: even if there were no other possible worlds, the internal necessity would still exist. This distinction is difficult to make within the dominant possible worlds approach to modalities and indeed it is common to hold that any metaphysically necessary proposition is also nomologically necessary (Shoemaker 1980, Fales 1993, Bird 2004). Drawing this distinction may seem odd to many readers but assimilating nomological necessity within the broader category of metaphysical necessity blurs an important distinction. What is central to nomological necessitation (rather than nomological necessity) is what brings about the occurrence of the nomologically necessitated event or state. Nothing brings about a metaphysically necessary proposition (or state) and so incorporating the nomological cases within the metaphysical case is inappropriate unless one is already committed to a Humean view within which no event brings about another event.

Tahko $(2015,520-521)$ explores the question of whether the possibility of laws changing over time sheds light on the possibility of them changing across possible worlds. A central example he considers is the possibility of the fine structure constant changing over time.16 Because the fine structure constant is defined in terms of other fundamental constants, so that a change in the former would require a change in at least one of the latter, laws in which one or more of those constants occur are possibilities for changing laws. ${ }^{17}$ In the other direction, what would the contingency of some fundamental laws say about the possibility of a law changing over time? Recall that Hooke's Law is a linear approximation to the actual behavior of wires, but one that is a good approximation over a specifiable interval of elongation. Call the actual law in that spatial interval $L_{1}$. We also know that a wire is still a wire in a larger spatial interval where the law takes on some nonlinear functional form $\mathrm{L}^{*}$ (and before the wire deforms beyond the point where a restorative force is present.) Despite the standard restriction that laws should be universal, there are often scope restrictions on the quantifiers regarding constraints on the domain of application of the law. Here there is a broad scope limitation to the region of elasticity and a tighter scope restriction to whatever the contingent limits of extension are within which Hooke's Law holds. If we allow such

\footnotetext{
${ }^{16}$ Takho's paper also arrives at the conclusion that there are some metaphysically necessary laws and some metaphysically contingent laws, but using an ontology of fundamental natural kinds to argue for that conclusion.

17 As of now, the evidence for temporal or spatial variations in the fine structure constant is inconclusive.
} 
restrictions on the scope of a law, why not permit temporal restrictions? We do not have at present a definitive answer to that question, but one way to approach it is to take the fact that in early epochs of the universe, decreases in temperature act as a surrogate for intervals of time. The question then becomes why restricting a fundamental law to certain regions of temperature is inadmissible. The standard response to that question is to invoke a unification principle, but absent a convincing metaphysical argument that the nomological structure of the universe must be simple, unification is merely a methodological principle that leaves open the possibility of temporally restricted laws.

\section{Changing Laws in Humeanism}

In the present section, we turn to Humean regularity theories about laws, which, contrary to the views discussed above, eschew any commitment to natural necessity. In particular, we discuss the extent to which Lewis-style 'best system analysis' could be amended in order to leave room for changing laws.

\subsection{Stage Setting}

Humeanism can be defined through the conjunction of two claims: (a) truth supervenes on being and (b) there are no necessary connections between distinct existents. The so-called 'Humean Mosaic' (hereafter HM; Lewis 1986, p.ix-x) allows for having a grasp on what exactly 'being' or 'existents' refer to: the world just is an arrangement of local, particular facts (typically space-time points having intrinsic qualities and related to one another by spatio-temporal relations). It is also a typical feature of Humeanism that existents that are not themselves part of HM nonetheless supervene on it, to the effect that everything that is not itself an arrangement of qualities only exists in virtue of some of HM's arrangements of qualities.

Given its rejection of necessary connections, Humeanism is very hospitable to regularity accounts of laws. The most sophisticated variant of these currently is the Mill-Ramsey-Lewis or Best System Account (hereafter BSA), according to which laws are those worldly regularities that are captured by the axioms and theorems of the true deductive system that best systematizes the world. Typically, a given system's degree of success in systematizing the world is dependent on a balance between the system's strength (the amount of information it gives about the world), 
simplicity (the syntactical complexity/number of its axioms) and fit (a measure of the likelihood of the world being as the system tells it is).

As they are not the kinds of things that directly belong to HM, BS-laws can only be said to exist insofar as they supervene on $\mathrm{HM}$, giving sense to the idea that, with respect to the BSA, the laws do not fix-nor do they govern, necessitate or bring about-the facts. Rather, they are what they are in virtue of some set $\mathrm{S}$ of facts of $\mathrm{HM}$. The exact nature of $\mathrm{S}$ is of course partly the topic of the present section. Typically-but, as we would like to claim in what follows, not necessarily$\mathrm{S}$ is taken to be the whole of $\mathrm{HM}$, comprising past, present and future facts throughout space. As it appears, the BSA is a reductive account of some sort: laws of nature reduce to a given set of non-nomic facts.

In order to allow for a comparison between the degrees of success of different deductive systems in systematizing our world, it proves necessary for these systems to be expressed into a unique language, which picks out those properties that happen to 'carve nature at its joints'. Such properties, deemed 'perfectly natural' (hereafter PN; Lewis 1983; 1986), are of a sparse and elite kind. They are the ones whose instantiations form the bedrock ontology of Humeanism - the very 'tiles' of HM-so that they constitute the ultimate supervenience basis for all the other, non-PN properties. Though the very distinction between $\mathrm{PN}$ and non-PN properties is to be taken as primitive, PN properties are the ones which ultimately figure into the laws, and it is up to science to tell us - always only tentatively to be sure-which are the PN properties of our world.

Typically, mainstream Humeanism has the following features: 18

(i) Fundamentalism: PN properties form a unique set of properties whose instantiations constitute the bedrock ontology of a Humean world, viz. HM (Lewis 1994a). From such a bedrock, other, derivative existents obtain through supervenience. Fundamental laws of nature, as axioms of the best system, only refer to PN properties.

(ii) Physicalism: PN properties are physical and should therefore be identifiable as such by (the final) physics (Lewis 1994a).

\footnotetext{
18 We could have added realism to the list, insofar as, primarily, BS-laws are supposed to be things in the world, namely regularities or patterns among facts of $\mathrm{HM}$. It is only secondarily that they are captured by formal devices that allow for organizing knowledge of the world (Earman 1984; Ramsey's epistemological version of the BSA is perhaps a notable exception to this).
} 
(iii) (Mereological) reductionism: Non-PN properties reduce to PN properties. Such a reductionism immediately follows from a strong interpretation of supervenience (Lewis 1994b), to the effect that emergent properties are ipso facto to be excluded (Lewis 1986). ${ }^{19}$

(iv) Eternalism: Past, present and future facts are ontologically on a par, to the effect that there is no genuine becoming in typical Humean worlds. This usually translates as considering $\mathrm{HM}$ as the block universe (Friebe 2018).

(v) Optimism: In some way, 'nature is kind to us', to the effect that there is only one deductive system that comes out best of the competition for lawhood (Lewis 1994a). Such an assumption is the Humean surrogate for Armstrong- or Ellis-style timeless necessary connections for the purpose of mitigating the skeptical problem of induction (Beebee 2011).

Though crucial, feature (v) is, as such, obviously very vague. The typical way to make it precise is to translate it into a commitment about the putative universality of BS-laws, as follows:

$\left(v^{*}\right)$ Universalism: BS-laws hold everywhere and everywhen. They are regularities that span-or their supervenience basis $S$ is - the whole of $\mathrm{HM}$. This precludes them from having exceptions (Lewis 1986; or Swartz 2003, chapter 9).

Together with the defining ideas (a) and (b) of Humeanism, features (i)-( $\left.\mathrm{v}^{*}\right)$ delineate the canonical, Lewis-style BSA package. Heterodox versions of the account have recently flourished, which cast doubt upon the necessity-or even the very soundness - of some of these features. Though they all converge on rejecting ( $\left.v^{*}\right)$, 'Better Best System Accounts' challenge (i)-(iii), in the spirit of a non-fundamentalist, non-physicalist and antireductionist version of the BSA (Schrenk 2008; Cohen \& Callender 2009), whereas varieties of 'open-future Humeanism' tend rather to put (iv) into question (Backmann 2016; Smart 2018).

\footnotetext{
19 This is true with respect to traditional, synchronic emergence. Humeanism is actually more tolerant with diachronic emergence (Sartenaer 2019).
} 
In a more conservative spirit, we would like to question here the necessity of building ( $\left.\mathrm{F}^{*}\right)$ into the BSA without having to go as far as to give up on any of the claims (i)-(v). To be sure that BS-laws are universal (that is, omnispatial and omnitemporal) is a powerful intuition that is prima facie well-motivated. It appears indeed that the wider the spatial and temporal extension of laws is, the more probable it is that they score high in strength at a low cost to simplicity. After all, best systems presumably are the ones with few but powerful axioms. Though sound, such a motivation should nonetheless not lead us to consider ( $\left.\mathrm{V}^{*}\right)$ as a sine qua non condition for BSlawhood (Earman 1978). As we show below, it could well be that general statements with some restricted scope-or general statements supervening on a set of facts S smaller than the whole of $\mathrm{HM}$-turn out to be powerful and simple enough (in comparison with more general statements) for finding their way into the best system.

\subsection{Against (v*): BS-Laws Can Change}

In this section, we show that there are ways in which $\left(v^{*}\right)$ breaks down that nonetheless allow for the competition between deductive systems to designate a unique, best system of laws, provided that nature remains, to some extent, kind to us. Two of these ways correspond to scenarios where BS-laws admit few, local violations, and where BS-laws change in a particular way. But beforehand, a preliminary definition:

A given world is (BS-)law-based as soon as, at the end of time, it is possible to unambiguously designate a unique best system (insofar as such a system strikes the best balance, by far, between simplicity, strength and fit).

Let us first consider a world $\mathbf{W}$ that is an infinitely extended Euclidean space-time containing $n$ massive point-like particles. Together with a specification of the particles' masses, positions and velocities at any given time, the particles' trajectories can be fully recovered from a unique general statement $L$ (say, in a Newtonian spirit, $\mathbf{F}=G m m^{\prime} r^{-2} \mathbf{u}$ ). Surely, $\mathbf{W}$ is law-based. A competition run against all the deductive systems that are true to $\mathbf{W}$, and which only pick out its 
PN properties (here mass), is going to unambiguously designate a unique best system, namely $\{F t$, $L$ \}, with $F^{t}$ being a factual statement about the physical state of $\mathbf{W}$ at $t .^{20}$

Now consider a set of worlds $\mathbf{W}_{n}$, which are similar to $\mathbf{W}$ except from the fact that, in $n$ infinitesimal portions of spacetime, $L$ fails to be true. Recent works in the Humean tradition tend to indicate that, in spite of the existence of such 'violations' of $L$, worlds like this can happen to be law-based provided that, among other things, $n$ is small. 'Lossy laws' are indeed not to be excluded a priori from the BSA, notably because the sheer amount of informativeness they carry with them is not always outbalanced by the drop in simplicity that any ceteris paribus clause inexorably induces in a deductive system (Braddon-Mitchell 2001; Schrenk 2014). ${ }^{21}$ This is certainly true, for instance, for world $\mathbf{W}_{1}$, where only one local 'violation' of $L$ is supposed to occur; its best system therefore being $\left\{F^{t}, S_{1}, L\right\}$, where $S_{1}$ is a statement about the domain of validity of $L$ or the portion of $\mathbf{W}_{1}$ 's HM on which $L$ supervenes (being the whole of HM minus the only infinitesimal 'exception'). It is notable that, already in $\mathbf{W}_{1}, L$ is not universal, although it is invariant in its own domain, in a way that is respectful of the regularist spirit of the BSA. As it appears, the very fact that there exist portions of a world's HM that its laws fail to systematize is not necessarily enough for depriving these laws from being the best handles for systematizing knowledge about this world.

Let us now turn to worlds in which $n$ takes on an infinite value, that is, worlds in which $L$ has an infinite amount of 'violations'. With respect to such worlds, two distinct cases are worth considering. First, L's violations could be scattered randomly and discretely throughout spacetime. In such dramatically 'unkind' situations, it is plausible that adding a ceteris paribus clause to $L$ for each of its 'violations' will add too much complexity for it to deserve its entry into the best system. It is even plausible that the corresponding worlds would fail to be law-based at all. Secondly and by contrast, it could also be that $L$ 's violations are themselves regular, so that they

\footnotetext{
${ }^{20}$ When $t$ is set up at the origin of W, Ft captures W's initial conditions. As it appears, we are inclined to stick to Lewis' letter here, by (i) allowing for factual statements to figure into the best system - for they certainly provide the system with a boost in strength against a not too drastic sacrifice in simplicity-and (ii) not considering these statements as lawful (Lewis 1983, p.367). Recent Lewisian thinkers are prone to accept (i) but deny (ii), that is, they consider statements of facts about a world's initial conditions as worthy of figuring in the best system while being themselves lawful (Albert 2000; Loewer 2012). Though it is a matter of possible controversy that the BSA actually has the resources to draw a distinction between laws and boundary conditions (Hicks 2018), this will not keep us busy any further here. It should also be noted that the virtue of fit is left aside in the present discussion as the worlds at stake are deterministic.

${ }^{21}$ At some point, Lewis himself seemed to have endorsed such a view in spite of the supposed universality of BS-laws (Lewis 1973, p.75).
} 
can be expressed through a highly compressed shortcut that itself deserves its entry into the best system. A case like this would be a world $\mathbf{W}_{c}$ where $L$ is true everywhere up to time $t_{c}$, after which $L^{*}$ (say $\mathbf{F}=-G m m^{\prime} r^{-2} \mathbf{u}$ ) becomes true. Using a canonical metaphor, in order to give scientists knowledge about such a world, God would not need to give them $L$ and an infinite compendium of all its 'violations', nor would She need to provide $L^{*}$ and an infinite compendium of all its 'violations', for $L$ and $L^{*}$ would themselves be informationally redundant with these compendia. All that would be needed is - that is, $\mathbf{W}_{c}$ 's best system would be $-\left\{F^{t}, S, S^{*}, L, L^{*}\right\}$, where the factual information about the 'turning point' between $L$ and $L$ 's regimes is contained within the specification of their supervenience bases $S$ and $S^{*}$.

The main morale of this discussion is this: if ceteris paribus laws can be Humeanly kosher, so can changing laws. The situation in which both kinds of law happen to figure in a world's best system is the same: the complexity that is added by the factual statements necessary to pin down the laws' domains of validity (or supervenience basis) must be outbalanced by the informativeness of these laws. Though for some worlds it can be impossible to figure out whether such a situation is actually the case, in relatively kind worlds, such as $\mathbf{W}_{1}$ and $\mathbf{W}_{c}$ where information about the laws' 'violations' is either very limited or highly compressible, e.g., for it is itself in accordance with a law, non-universal, yet-invariant-in-their-domain laws deserve their entry into the best systematization of the world.

\subsection{Changing Laws and HM's Nomological Structure}

As it appears, in order for BS-laws to change in a given world, the world's HM has to possess some primitive structure-let us call it the 'nomological structure'-in the sense that it has to be chopped into spatiotemporally distinct regions, each of which is made of a different pattern of instantiations of PN properties and amenable to its own separate best system analysis (leading to a different system of laws). ${ }^{22}$ That a world's Humean mosaic possesses such a nomological structure is ultimately apparent in its overall best system, as encoded into the distinct domains of validity $S^{i}$ of its laws $L^{i}$. It could even be that, from an epistemological point of view, it

\footnotetext{
22 Although in the example of world $\mathbf{W}_{c}$ the nomological structure is purely temporal, it could well be (rather or also)
} spatial. 
is only through a world's overall best system that we can come to know what that world's nomological structure happens to be.

Of course, one could be reluctant to build some hidden structure into HM, insofar as, prima facie, doing so tends to conflict with the typical ontological sobriety of Humeanism. However, it is already the case in mainstream, Lewisian Humeanism that the very nature of a world's HM is in itself brute and primitive (as well as contingent, although what natural properties there are is supposed to hold with metaphysical necessity). 'If one is a Humean, then the Humean Mosaic itself appears to admit of no further explanation' (Maudlin 2007, p.172). Accordingly, unless primitiveness happens to be a matter of degree-which we think it is not-a Humean mosaic with an intricate nomological structure is not more mysterious or unanalyzable that a Humean mosaic with the most degenerate nomological structure there is, viz. a unique domainactually the whole of $\mathrm{HM}$-that is the supervenience basis for all the (then immutable) laws of nature.

In light of this, here is a definition: a law $L$ is a changing BS-law of world $\mathbf{W}$ iff:

(i) $L$ is a BS-law of $\mathbf{W}$, that is, it appears in the (non-factual part of) its best system, and

(ii) $L$ is changing, that is, there is at least one best sub-system of $\mathbf{W}$ in which a surrogate $L^{*}$ of $L$ appears in place of $L$.

By a 'surrogate' law $L^{*}$, we mean a law that systematizes - or a worldly regularity among instances of the very same PN properties as $L$, and which cannot be subsumed under $L$ (for example, in world $\mathbf{W}_{c}$, both $L$ and $L^{*}$ describe distinct ways in which instances of mass are patterned across HM).

Accordingly, a changing law $L$ is a law that is neither universal nor merely ceteris paribus (it is only 'violated' in a way that is itself BS-lawful). In a world $\mathbf{W}$ containing a changing law $L$, there is always at least one region $S$ of $\mathrm{HM}$ in which $L$ is a law-insofar as $L$ is a particularly efficient handle to systematize knowledge about $S$ - and one region $S^{*}$ in which $L$ is not a law, to the effect that there is a surrogate $L^{*}$ of $L$ that turns out to be a better handle than $L$ for systematizing 
knowledge about $S^{*}$. Ultimately, that $L$ is a law in $S$ but not in $S^{*}$ is dependent on the way in which the instances of the PN properties are patterned, as it should be ${ }^{23}$.

\section{Conclusion}

In this paper, we aimed at answering the question-with respect to three of the most widespread theories of laws of nature available-what price changing laws of nature?

In section 1, which was devoted to Armstrong's necessitarianism, we argued that the most cost-effective way of accommodating changing laws was to weaken the very notion of a universal. In particular, we have shown that changing laws could fall squarely within such a governist framework as soon as one is ready to build spatial or temporal restrictions into the universals themselves, in the spirit of Armstrong's own concept of quasi-universal. Though open, such an option would nonetheless need to be fully fleshed out, notably by devising a (currently non-existing) theory of indexical universals.

We then provided an argument in section 2 to the effect that dispositionalism should not necessarily be tied to essentialism, by showing that some properties can happen to be identified through criteria that are independent from the manifestations of their dispositions. Incidentally, this entailed that properties can well be identified without any explicit reference to the laws covering the possible manifestations of their dispositions (and, a fortiori, to these laws' exact mathematical form). Such an observation opens the door for a nonessentialist version of dispositionalism which allows for (at least some) dispositions to change over time (and, with them, the laws).

Finally, in section 3, we turned to Humeanism and Lewis' 'best system' take on laws of nature. We argued that the only reason why best system laws could be seen as immutable is because the supervenience basis of all these laws has been set up 'by hand' to the whole spatiotemporal extension of the Humean mosaic (in the spirit of making sure that nomic regularities are

\footnotetext{
23 It has recently been suggested that one way of breaking free of the immutability of BS-laws is to embrace a growing block universe ontology (see for instance Backmann's (2016) 'open-future Humeanism' or Smart's (2018)

'hypertemporal Humeanism'). Even more, the resulting account of changing BS-laws would be 'true[r] to Hume' (Smart 2018, p.109). Though we are of course sympathetic to these endeavors, we also think that their commitment to a growing block universe ontology is (i) risky-for the combination of the view with Humeanism is notoriously unstable (Hüttemann 2014; Briggs \& Forbes 2017)-and, more importantly perhaps, (ii) unnecessary for the sole sake of securing the laws' mutability. As we have seen, it is indeed enough for BS-laws to change that there are adjacent regions of HM (as a block universe) in which the laws differ (especially when the relation of adjacency can be purely temporal, as in the case of $\mathbf{W}_{c}$ ). As such, it appears an unnecessary metaphysical inflation to consider that changing BS-laws must also pop into existence at a certain turning point in time.
} 
robust enough for our world to be law-based). Removing such an undue restriction allowed the relaxation of the account in order for it to accommodate ceteris paribus as well as changing laws, without having to tamper with its core defining features.

As it appears, necessitarianism, dispositionalism and Humeanism do not cope in the same way with the possibility of changing laws. While the latter only needs superficial adjustments that would not be considered as too heterodox by its own proponents, both necessitarianism and dispositionalism have to make some bigger concessions. It remains to be seen whether or not this would count as a flaw, depending on whether or not the very idea of laws changing appears to gain plausibility over the years.

\section{References}

Albert, D. Z. (2000). Time and Chance. Cambridge (Mass.): Harvard University Press.

Armstrong, D. M. (1983). What Is a Law of Nature? Cambridge: Cambridge University Press.

Armstrong, D. M. (1989). Universals, An Opinated Introduction. Boulder: Westpress View.

Armstrong, D. M. (1997). A World of States of Affairs. Cambridge: Cambridge University Press.

Backmann, M. (2016). I Tensed the Laws and the Laws Won: Non-Eternalist Humeanism.

Manuscrito, 39(4), 255-277.

Balashov, Y. V. (1992). On the Evolution of Natural Laws. The British Journal for the Philosophy of Science 43(3): 343-370.

Beebee, H. (2011). Necessary Connections and the Problem of Induction. Noûs, 45(3), 504-527.

Bird, A. (2004). Strong Necessitarianism: The Nomological Identity of Possible Worlds. Ratio 17(3): 256-276.

Bird, A. (2007). Nature's metaphysics: Laws and properties. Oxford: Oxford University Press.

Braddon-Mitchell. (2001). Lossy Laws. Noûs, 35(2), 260-277.

Brading, K. (2013). Three Principles of Unity in Newton. Studies in History and Philosophy of Science 44(3): 408-15.

Briggs, R., and Forbes, G. A. (2017). The Growing-Block: Just one Thing after Another? Philosophical Studies 174: 927-943.

Cartwright, N., and Merlussi, P. (2018). Are Laws of Nature Consistent with Contingency? In W. Ott, and L. Patton (Eds.), Laws of Nature, Oxford: Oxford University Press.

Cohen, J., and Callender, C. (2009). A Better Best System Account of Lawhood. Philosophical Studies 145(1): 1-34. 
Dirac, P. A. M. (1937). The Cosmological Constant. Nature 13: 323-324.

Earman, J. (1978). The Universality of Laws. Philosophy of Science 45(2): 173-181.

Earman, J. (1984). Laws of Nature: The Empiricist Challenge. In R. J. Bogdan (Ed.), D. M. Armstrong (pp. 191-223). Dordrecht: Springer Verlag.

Earman, J. (2004). Laws, Symmetry, and Symmetry Breaking: Invariance, Conservation Principles, and Objectivity. Philosophy of Science 71(5): 1227-1241.

Ellis, B. (2001). Scientific Essentialism. Cambridge: Cambridge University Press.

Fales, E. (1993). Are Causal Laws Contingent? In J. Bacon, K. Campbell, and L. Reinhardt (eds), Ontology, Causality, and Mind (pp. 121-144). Cambridge: Cambridge University Press.

Fine, K. (2002). The Varieties of Necessity. In T. Gendler, and J. Hawthorne (eds.), Conceivability and Possibility (pp. 253-281). Oxford: Oxford University Press.

Friebe, C. (2018). Metaphysics of Laws and Ontology of Time. Theoria 33(1): 77-89.

Hendry, R. F., and Darrell P. R. (2009). Dispositional essentialism and the necessity of laws. Analysis 69: 668-677.

Hicks, M. T. (2018). Dynamic Humeanism. The British Journal for the Philosophy of Science 69(4): 983-1007.

Hildebrand, T. (2018). Natural Properties, Necessary Connections, and the Problem of Induction. Philosophy and Phenomenological Research 96(3): 668-89.

Hüttemann, A. (2014). Scientific Practice and Necessary Connections. Theoria, 29(79): 29-39.

Keinänen, M. (2014). Tropes, Causal Processes, and Functional Laws. In S. Miroslaw and M. Rosiak (Eds.), Substantiality and Causality (pp. 35-50). Berlin: De Gruyter.

Lange, M. (2009). Could the Laws of Nature Change? Philosophy of Science 75: 69-92.

Lewis, D. (1973). Counterfactuals. Oxford: Basil Blackwell.

Lewis, D. (1983). New Work for a Theory of Universals. Australasian Journal of Philosophy 61(4): 343-377.

Lewis, D. (1986). Philosophical Papers (Vol. II). Oxford: Oxford University Press.

Lewis, D. (1994a). Humean Supervenience Degugged. Mind 103(412): 473-490.

Lewis, D. (1994b). Reduction of Mind. In S. Guttenplan (Ed.), A Companion to Philosophy of Mind (pp. 412-431). Oxford: Blackwell Publishers.

Loewer, B. (2012). Two Accounts of Laws and Time. Philosophical Studies 160(1): 115-137.

Lowe, E. J. (2002). Kinds, Essence, and Natural Necessity. In. A. Bottani, M. Carrara and P. Ciaretta (Eds.), Individuals, Essence and Identity (pp. 189-206). Dordrecht: Springer.

Maudlin, T. (2007). The Metaphysics Within Physics. New York: Oxford University Press.

Poincaré, H. (1911). L'évolution des lois. Scientia 5(9): 275-292.

Sartenaer, O. (2019). Humeanism, Best System Laws, and Emergence. Philosophy of Science 86(4): 719)-738 
Schrenk, M. (2008). A Theory for Special Sciences Laws. In H. Bohse, K. Dreimann, and S. Walter (Eds.), Selected Papers Contributed to the Sections of GAP.6, 6th International Congress of the Society for Analytical Philosophy. Paderborn/Munster: Mentis.

Schrenk, M. (2014). Better Best Systems and the Issue of CP-laws. Erkenntnis 79(10): 1787-1799.

Shimony, A. (1999). Can the Fundamental Laws of Nature Be the Result of Evolution? In Butterfield, J., and Pagonis, C. (eds.), From Physics to Philosophy (pp. 208-223). Cambridge:

Cambridge University Press.

Shoemaker, S. (1980). Causality and Properties. In P. van Inwagen (ed.), Time and Cause: Essays Presented to Richard Taylor (pp. 109-135), Dordrecht: Reidel.

Smart, B. (2018). True-to-Hume Laws and the Open-Future (or Hypertemporal Humeanism). South African Journal of Philosophy 37(1): 99-110.

Smolin, L. (2015). Temporal Naturalism. Studies in History and Philosophy of Modern Physics 52: 86-102.

Swartz, N. (2003). The Concept of Physical Law. Cambridge: Cambridge University Press.

Swoyer, C. (1982). The Nature of Natural Laws. Australasian Journal of Philosophy 60: 203-223.

Tahko, T. E. (2015). The Modal Status of Laws: In Defence of a Hybrid View. The Philosophical Quarterly 65: 509-528.

Tugby, M. (2013). Graph-theoretic Models of Dispositional Structures. International Studies in the Philosophy of Science 27: 23-39.

Whitehead, A. N. (1933). Adventures of Ideas. Cambridge: Cambridge University Press.

Wigner, E. P. (1979). Symmetries and Reflections: Scientific Essays of Eugene P. Wigner. Reprint of 1967. Woodbridge, Connecticut: Ox Bow Press.

Yudell, Z. (2013). Lange's Challenge: Accounting for Meta-Laws. The British Journal for the Philosophy of Science 64 (2): 347-69. 